Article

\title{
A Lateral Flow Immunoassay for the Rapid Identification of CTX-M-Producing Enterobacterales from Culture Plates and Positive Blood Cultures
}

\author{
Sandrine Bernabeu ${ }^{1,2}$, Kayaththiry Caroline Ratnam ${ }^{2}$, Hervé Boutal ${ }^{3}$, Camille Gonzalez ${ }^{2}$, \\ Anaïs Vogel ${ }^{3}$, Karine Devilliers ${ }^{3}$, Marc Plaisance ${ }^{3}$, Saoussen Oueslati ${ }^{1,2}$, \\ Surbhi Malhotra-Kumar ${ }^{4,5}$, Laurent Dortet ${ }^{1,2,6} \mathbb{1}^{\circ}$, Nicolas Fortineau ${ }^{1,2,6}$, Stéphanie Simon ${ }^{3} \mathbb{C}^{\text {, }}$ \\ Hervé Volland ${ }^{3}$ and Thierry Naas $1,2,5,6,7, *$ (D) \\ 1 Team Resist, UMR1184, School of Medicine of Université Paris-Saclay-INSERM-CEA, LabEx Lermit, \\ 94276 Le Kremlin-Bicêtre, France; sandrine.bernabeu@aphp.fr (S.B.); oueslati.saoussen@gmail.com (S.O.); \\ laurent.dortet@aphp.fr (L.D.); nicolas.fortineau@aphp.fr (N.F.) \\ 2 Bacteriology-Hygiene Unit, APHP, Hôpital Bicêtre, 94270 Le Kremlin-Bicêtre, France; \\ ratnam.caroline@gmail.com (K.C.R.); gonzalezcamille0405@gmail.com (C.G.) \\ 3 Service de Pharmacologie et Immunoanalyse (SPI), CEA, INRA, Laboratoire d'Etudes et de Recherches \\ en Immunonalyse, Université Paris-Saclay, 91191 Gif-sur-Yvette, France; Herve.BOUTAL@cea.fr (H.B.); \\ anais.vogel@cea.fr (A.V.); karine.devilliers@cea.fr (K.D.); marc.plaisance@cea.fr (M.P.); \\ stephanie.simon@cea.fr (S.S.); herve.volland@cea.fr (H.V.) \\ 4 Laboratory of Medical Microbiology, Vaccine \& Infectious Disease Institute, University of Antwerp, \\ 2610 Antwerp, Belgium; surbhi.malhotra@uantwerpen.be \\ 5 Members of ESCMID Study Group for Antimicrobial Resistance Surveillance-ESGARS, Headquarter, \\ 4010 Basel, Switzerland \\ 6 Associated French National Reference Center for Antibiotic Resistance: Carbapenemase-Producing \\ Enterobacteriaceae, 94270 Le Kremlin-Bicêtre, France \\ 7 Service de Bactériologie, AP-HP, CHU de Bicêtre, 78 Rue du Général Leclerc, \\ 94275 Le Kremlin-Bicêtre, France \\ * Correspondence: thierry.naas@aphp.fr; Tel.: +33-1-45-21-29-86
}

Received: 16 September 2020; Accepted: 27 September 2020; Published: 28 September 2020

\begin{abstract}
We have developed a lateral flow immunoassay (LFIA), named NG-Test CTX-M MULTI (NG-Test), to detect group 1, 2, 8, 9, 25 CTX-M producers from agar plates and from positive blood cultures in less than $15 \mathrm{~min}$. The NG-Test was validated retrospectively on 113 well-characterized enterobacterial isolates, prospectively on 102 consecutively isolated ESBL-producers from the Bicêtre hospital and on 100 consecutive blood cultures positive with a gram-negative bacilli (GNB). The NG-Test was able to detect all CTX-M producers grown on the different agar plates used in clinical microbiology laboratories. No false positive nor negative results were observed. Among the 102 consecutive ESBL isolates, three hyper mucous isolates showed an incorrect migration leading to invalid results (no control band). Using an adapted protocol, the results could be validated. The NG-Test detected 99/102 ESBLs as being CTX-Ms. Three SHV producers were not detected. Among the 100 positive blood cultures with GNB tested 10/11 ESBL-producers were detected (8 CTX-M-15, 2 CTX-M-27). One SHV-2-producing-E. cloacae was missed. The NG-Test CTX-M MULTI showed $100 \%$ sensitivity and specificity with isolates cultured on agar plates and was able to detect $98 \%$ of the ESBL-producers identified in our clinical setting either from colonies or from positive blood cultures.
\end{abstract}

Keywords: CTX-M; ESBL; LFIA; rapid detection; blood culture 


\section{Introduction}

The production of $\beta$-lactamases is a commonly encountered mechanism of resistance in Gram-negative bacteria, especially Enterobacterales [1]. In healthcare settings, extended-spectrum- $\beta$-lactamase (ESBL)-producing Enterobacterales (ESBL-E) are of particular concern. The CDC has estimated that ESBL-Es account for $19 \%$ of healthcare-related infections annually and that infections involving by these bacteria are also associated with increased mortality and cost of care [2]. ESBLs limit treatment options because they hydrolyze most $\beta$-lactams, including penicillins and third-generation cephalosporins (e.g., ceftazidime), and carry many other resistance determinants leading to a multidrug-resistant (MDR) phenotype [1].

Within the ESBLs, the CTX-M family appeared in the early $80 \mathrm{~s}$ and represent now the most prevalent ESBLs worldwide. They are divided into five groups based on amino acid sequence identity: the CTX-M-1, CTX-M-2, CTX-M-8, CTX-M-9, and CTX-M-25 groups. In each group are included minor allelic variants differing one from the other by one or few amino-acid substitutions [3-5]. Since the first description, they disseminated worldwide, becoming the most prevalent ESBLs $[6,7]$. The CTX-M-15 variant from the CTX-M-1 group is considered as the most prevalent one in many parts of the world. It is supplanted by CTX-M-9-group variants in China, Japan, South-East Asia and Spain for example, where the variant CTX-M-14 is dominant [7]. They have disseminated among a wide range of clinical bacteria species. Although they are susceptible to inhibitors, infections caused by CTX-M-producing bacteria present limited options of treatments. This situation has led to an increasing use of carbapenems, followed by the emergence and spread of carbapenemase-producing strains $[7,8]$.

Enterobacterales expressing CTX-Ms are associated with higher rates of healthcare costs, morbidity and mortality [7-12]. Infection control strategies for ESBL-Es are based on rapid identification of carriers. In addition, infections caused by ESBL-Es present limited treatment options, thus leading to an increasing use of carbapenems as observed in many countries [13]. Several diagnostic tests already exist for the identification of CTX-M carriers in clinical setups. They are mainly based on phenotypic approaches with antimicrobial susceptibility using disc diffusion methods in presence and absence of $\beta$-lactamase inhibitors [14,15]. These conventional screenings are usually slow, requiring an over-night culture $(\mathrm{o} / \mathrm{n})$, are labor-intense and require expertise for interpretation. The molecular methods, either DNA microarray-based and/or nucleic acid amplification-based remain the gold standard for the detection of CTX-Ms and other ESBLs [14-22]. Many of these assays correspond to home-made PCR assays $[19,20]$, but several assays are commercially available for CTX-M detection alone [18], and others for detecting also the 5 main carbapenemases such as the Verigene ${ }^{\circledR}$ system (Luminex Corporation, Austin, TX, USA) and the FilmArray ${ }^{\circledR}$ system (bioMérieux, Marcy l'Etoile, France). The two latter may be used directly from patients' positive blood cultures [21,22]. However, these tests are usually slow (1-4 h), labor-intense, expensive, require specific equipment and trained staff in order to be implemented in any clinical laboratory. To meet current needs, antimicrobial drug resistance detection methods must be cheap (reduced cost of consumables and equipment) and easy to use (reduce technical complexity) for the end user. Lateral flow immunoassays (LFIAs) have shown their usefulness as confirmatory tests for antibiotic resistance mechanisms detection, especially for $\beta$-lactamases in gram-negatives [23-25]. They provide easy, rapid, and reliable results in a few minutes directly on colonies isolated on selective media or disk diffusion antibiogram [23-25]. The aim of the study was to develop a rapid LFIA, named NG-Test CTX-M MULTI, for the detection of the CTX-Ms belonging to the five groups directly from colonies isolated on different culture media widely used in routine laboratory and from positive blood cultures.

Here, we validated the NG-Test CTX-M MULTI LFIA retrospectively on 113 well-characterized enterobacterial isolates, and prospectively on 102 consecutively isolated ESBL-producers and on 100 consecutive blood cultures positive with a gram-negative bacilli (GNB). All the isolates expressing a CTX-M where perfectly detected within $15 \mathrm{~min}$, showing $100 \%$ sensitivity and specificity for the 5-groups of CTX-M $\beta$-lactamases. 


\section{Materials and Methods}

\subsection{Ethics Statement}

All experiments were performed in compliance with French and European regulations on the care of laboratory animals (European Community [EC] Directive 86/609, French Law 2001-486, 6 June 2001) and with the agreements (approved 6 January 2016) of the ethics committee of the Commissariat à 1'Energie Atomique (CEtEA “Comité d'Ethique en Expérimentation Animale" $\mathrm{n}^{\circ} 44$ ) no. 12-026 and 15-055 delivered to S. Simon by the French Veterinary Services and CEA agreement D-91-272-106 from the Veterinary Inspection Department of Essonne (France).

\subsection{Monoclonal Antibodies}

Biozzi mice (10 weeks old) were immunized with the recombinant CTX-M-15 (M1-L291), CTX-M-2 (Q29-F291), and CTX-M-14 (Q29-L291) ß-lactamases, as they are the most prevalent variants belonging to the three main groups of CTX-Ms (group 2, 9 and 1, respectively). These enzymes were expressed with the hexa-histidine tag on their $C$ terminus as previously described [24,25]. For each enzyme, 20 monoclonal antibodies (mAbs) were tested, and the pairs of antibodies showing the best limit of detection (LOD) for CTX-M-expressing bacteria were selected for the NG-Test CTX-M MULTI assay as previously described $[24,25]$. The 3 best pairs of monoclonal antibodies selected (one against each targeted group), when combined in a single test line, were able to detect all 5 groups of CTXMs due to cross-reactivity of some antibodies with the two other groups (group 8 and 25).

\subsection{NG-Test CTX-M MULTI Assay and Protocol}

The selected antibodies were produced on a large scale and provided to NG biotech (Guipry, France) for the development of the NG-Test CTX-M MULTI assay (Figure 1). Capture antibodies were immobilized on a unique test line. Isolates to be tested were grown overnight at $37^{\circ} \mathrm{C}$ on $\mathrm{MH}$ agar plates. Using a $1 \mu \mathrm{L}$ inoculation loop, a single colony was resuspended in 5 drops (c.a. $100 \mu \mathrm{L}$ ) of the extraction buffer (lysis step) and vortexed a few seconds. Subsequently, $100 \mu \mathrm{L}$ of this extract were dispensed on the cassette, and the migration was allowed for $15 \mathrm{~min}$. The results were eye read by monitoring the appearance of a red band on the test line, along with a band corresponding to the internal control.

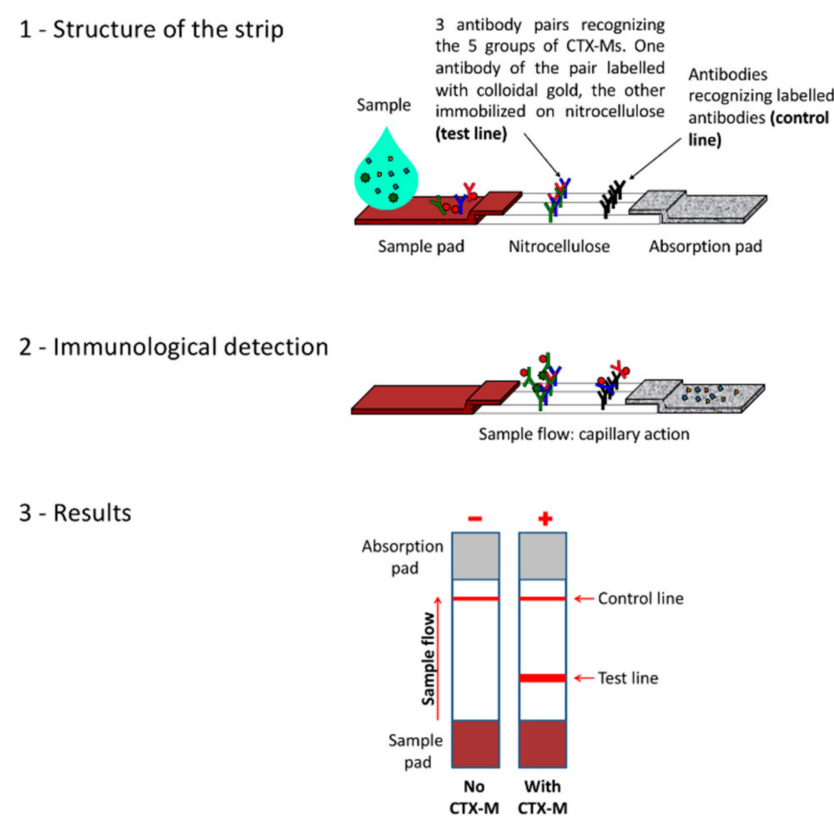

Figure 1. Principle of the assay. 


\subsection{Bacterial Strains}

For the retrospective validation, 113 enterobacterial isolates with PCR-characterized $\beta$-lactamase content were used to evaluate NG-Test CTX-M MULTI. This collection represented 13 non-CTX-M ESBL-producers, 51 CTX-M ESBL-producers, 28 Kluyvera spp and 21 Klebsiella oxytoca isolates. Twenty-seven different CTX-M variants were tested (CTX-M-1, -2, -3, -8, -9, -10, -13, -14, -15, -17 -18, $-19,-24,-27,-32,-37,-55,-57,-65,-71,-82,-93,-94,-100,-101,-127$, and 182) belonging to the CTX-M-1 group $(n=32)$, CTX-M-2 group $(n=2)$, CTX-M-8 group $(n=2)$, CTX-M-9 group $(n=13)$ and CTX-M-25 group $(n=2)$.

For the prospective evaluation, 102 enterobacterial clinical isolates showing an antibiotic susceptibility profile compatible with the expression of an ESBL (Resistance to expanded-cephalosporins, recovery with class A inhibitors, with or without a synergy image), were tested from MH-agar plates.

\subsection{Positive Blood Cultures, Isolate Identification and Susceptibility Testing}

100 consecutive blood cultures positive with Gram-negative bacteria as revealed by Gram staining collected between November 2018 and April 2019 were directly tested according to the protocol described by Takissian et al. [23]. Bacteria recovered from these blood cultures were identified using matrix-assisted laser desorption ionization-time of flight mass spectrometry (MALDI-TOF MS) with the Bruker MS system (Bruker Daltonics, Bremen, Germany), according to the manufacturer's instructions. Susceptibility testing was performed by the disk diffusion method on Mueller-Hinton agar plates (Bio-Rad, Marnes la Coquette, France) incubated for $18 \mathrm{~h}$ at $37^{\circ} \mathrm{C}$ and interpreted according to EUCAST guidelines [26].

\subsection{Resistance Gene Detection}

Genes coding for Ambler class A $\beta$-lactamases were sought by PCR using primers specific for the $b l a_{\mathrm{TEM}}, b l a_{\mathrm{SHV}}$, and bla $a_{\mathrm{CTX}-\mathrm{M}}$ genes, as previously described $[19,20]$. PCR products were purified using the QIAquick PCR purification kit (Qiagen, Courtabœuf, France) and sequenced on both strands with an automated sequencer (ABI 3100; Applied Biosystems, Foster City, CA, USA).

\subsection{Culture Media Tested}

Eleven representative isolates were grown on 5 commonly used culture media: Mueller-Hinton $(\mathrm{MH})$ agar (Biorad, Marnes la Coquette, France), URISelect 4 (Uri-4; Biorad, Marnes la Coquette, France), Columbia agar plus 5\% horse blood (COH; bioMérieux, Marcy l'Etoile, France), ChromID ESBL agar (bioMérieux, Marcy l'Etoile, France) and Drigalski agar (bioMérieux, Marcy l'Etoile, France).

\section{Results}

\subsection{Retrospective Evaluation on Well-Characterized Isolates}

NG-Test CTX-M-MULTI was able to detect unambiguously all the 51 CTX-M-producing isolates including 32 CTX-M-1-group (i.e., CTX-M-1, -3, -10, -15, -32, -37, -55, -57, -71, -82, -101, -127 and -182 enzymes), 2 CTX-M-2, 2 CTX-M-8-group, 13 CTX-M-9-group (i.e., CTX-M-9, -13, -14, -17, -18, -19, $-24,-27,-65$ and -93 enzymes) and 2 CTX-M-25-group (e.g., CTX-M-94 and -100 enzymes) producers (Figure 2 and Table S1). The detection occurred equally well with the different Enterobacterales species tested (30/30 E. coli, 5/7 K. pneumoniae, 4/4 E. cloacae, 2/3 C. freundii, 2/2 K. oxytoca, 2/2 P. mirabilis, 1/1 C. koseri). For three mucoid bacterial isolates (2 K. pneumoniae and $1 \mathrm{C}$. freundii) an invalid test result was obtained, as revealed on the strip by the absence of a materialized control line (Figure $2 \mathrm{~g}$ ). For these isolates, one colony was resuspended in extraction buffer, vortexed vigorously for $3 \mathrm{~min}$, and incubated for $10 \mathrm{~min}$ at room temperature prior to loading on the LFIA. Using this adapted protocol, the three isolates were efficiently detected as CTX-M positive. The 13 isolates expressing non-CTX-M ESBL 
including 3 SHV-like, 6 TEM-like, 1 VEB-like and 3 SHV-like and TEM- like co-producers gave negative results (Table S1).

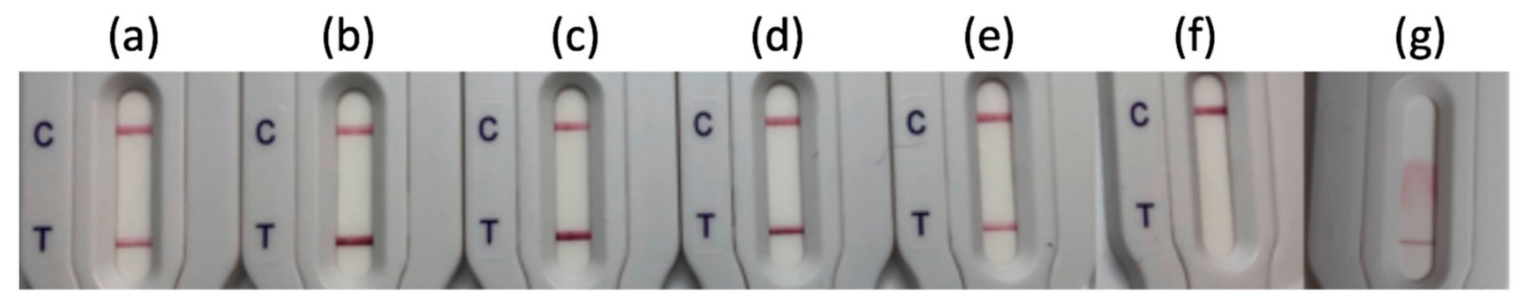

Figure 2. NG-Test CTX-M-MULTI results obtained with (a) E. coli expressing CTX-M-15; (b) E. coli expressing CTX-M-2; (c) E. coli expressing CTX-M-8; (d) E. coli expressing CTX-M-14; (e) E. coli expressing CTX-M-100; (f) E. cloacae expressing SHV-12 and (g) K. pneumoniae expressing CTX-M-18 that yeilded an invalid test result. One colony resuspended in extraction buffer and loaded on the cassette as recommended by the manufacturer. $\mathrm{C}$ stands for control line and $\mathrm{T}$ for test line.

As K. oxytoca produces a chromosomally encoded B-lactamase that is closely related to CTX-Ms in terms of AA sequence identity ( $88 \%$ AA identity), we tested 21 clinical $K$. oxytoca isolates expressing various acquired B-lactamases in addition to the endogenous OXY variant (Figure 3; Table S2). None of the K. oxytoca isolates tested gave a positive test result, except four isolates that produced an acquired plasmid-encoded CTX-M-15. Finally, as CTX-M enzymes derive from chromosomally borne $\beta$-lactamases from different Kluyvera species, 28 Kluyvera spp. isolates were tested, being either reference isolates of the Pasteur Institute strain depository (CIP) or clinical isolates from our strain collections. Thus $3 \mathrm{~K}$. cryocescens, 1 Kluyvera sp., $3 \mathrm{~K}$. cochlae, $1 \mathrm{~K}$. georginia, and $20 \mathrm{~K}$. ascorbata were tested (Figure 3; Table S3). One K. ascorbata gave a strong result, which is correlated with the high level of resistance to $\beta$-lactams of this isolate (Data not shown). Interestingly some isolates (8) had very faint bands, suggesting low level expression, while 19 gave a clear negative result, compatible with the fact that in Kluyvera spp., the chromosomally encoded CTX-M variants are weekly, if at all, expressed.

(a)

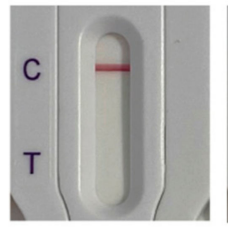

(e)

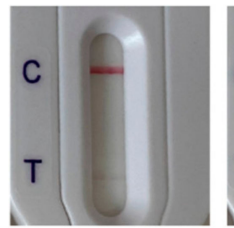

(b)

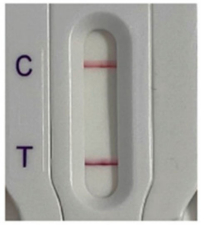

(f)

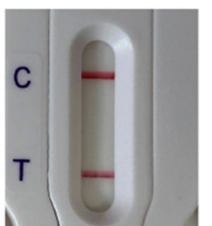

(c)

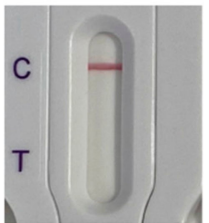

(g)

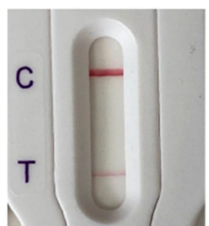

(d)

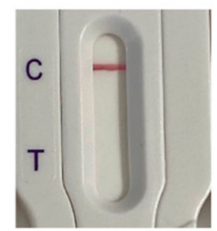

(h)

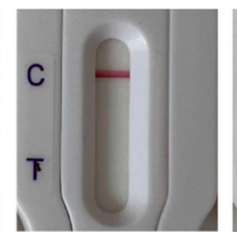

(i)

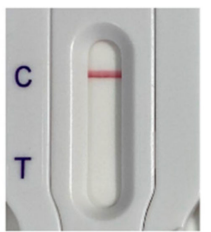

Figure 3. NG-Test CTX-M-MULTI results obtained with (a) K. oxytoca expressing NDM-1; (b) K. oxytoca expressing OXA-48, VIM-1 and CTX-M-15; (c) K. oxytoca WT; (d) K. oxytoca expressing TEM-1; (e) K. ascorbata CIP8295; (f) K. ascorbata (O23C8; patient); (g) K. ascorbata (O38I1, patient); (h) K. cryocrescens (CIP7952); (i) K. ascorbata (20-141, patient). One colony resuspended in extraction buffer and loaded on the cassette as recommended by the manufacturer. $\mathrm{C}$ stands for control line and $\mathrm{T}$ for test line. 
Taken together the NG-Test CTX-M MULTI is a highly sensitive and specific assay (as no false positive nor false negative results were observed) for CTX-M detection.

\subsection{Evaluation of NG-Test CTX-M MULTI Results on Different Culture Media}

Eleven isolates (2 CTX-M-1 group, 2 CTX-M-2-group, 2 CTX-M-8 and 2 CTX-M-9-group producers and 3 non-CTX-M producers) from the retrospective validation assay were grown on 5 of the most commonly used media for bacterial growth (Table 1). Some media currently used for the identification and/or selection of ESBL-expressing strains generate colonies with genus-specific colors (blue, green, pink, or dark purple on Uri-4 plates for example). These colored colonies, once suspended in the extraction buffer, stained the latter in a similar manner. This staining didn't interfere with the results. The 8 CTX-M-expressing strains gave positive results and the 3 non-CTX-M-producers gave negative results. Colony staining didn't change the appearance of the nitrocellulose membrane and still yielded easily interpretable results (data not shown).

Table 1. Evaluation of NG-Test CTX-M MULTI results on different culture media.

\begin{tabular}{ccccccc}
\hline & & \multicolumn{5}{c}{ Test Results on Culture Medium } \\
Bacterial Species & $\beta$-Lactamase & MH & COH & Uri-4 & DRIG & ChromID \\
\hline K. pneumoniae & CTX-M-15 & $\mathrm{P}$ & $\mathrm{P}$ & $\mathrm{P}$ & $\mathrm{P}$ & $\mathrm{P}$ \\
E. coli & CTX-M-1 & $\mathrm{P}$ & $\mathrm{P}$ & $\mathrm{P}$ & $\mathrm{P}$ & $\mathrm{P}$ \\
E. coli & CTX-M-14 & $\mathrm{P}$ & $\mathrm{P}$ & $\mathrm{P}$ & $\mathrm{P}$ & $\mathrm{P}$ \\
E. coli & CTX-M-9 & $\mathrm{P}$ & $\mathrm{P}$ & $\mathrm{P}$ & $\mathrm{P}$ & $\mathrm{P}$ \\
E. coli & CTX-M-8 & $\mathrm{P}$ & $\mathrm{P}$ & $\mathrm{P}$ & $\mathrm{P}$ & $\mathrm{P}$ \\
K. pneumoniae & CTX-M-8 & $\mathrm{P}$ & $\mathrm{P}$ & $\mathrm{P}$ & $\mathrm{P}$ & $\mathrm{P}$ \\
E. coli & CTX-M-2 & $\mathrm{P}$ & $\mathrm{P}$ & $\mathrm{P}$ & $\mathrm{P}$ & $\mathrm{P}$ \\
K. pneumoniae & CTX-M-2 & $\mathrm{P}$ & $\mathrm{P}$ & $\mathrm{P}$ & $\mathrm{P}$ & $\mathrm{P}$ \\
E. coli & SHV-12 & $\mathrm{N}$ & $\mathrm{N}$ & $\mathrm{N}$ & $\mathrm{N}$ & $\mathrm{N}$ \\
E. coli & TEM-24 & $\mathrm{N}$ & $\mathrm{N}$ & $\mathrm{N}$ & $\mathrm{N}$ & $\mathrm{N}$ \\
E. coli & KPC-2 & $\mathrm{N}$ & $\mathrm{N}$ & $\mathrm{N}$ & $\mathrm{N}$ & $\mathrm{N}$
\end{tabular}

a $\mathrm{MH}$, Mueller Hinton agar; $\mathrm{COH}$, Columbia agar plus 5\% horse blood; Uri-4, URISelect 4 agar medium; DRIG, Drigalski agar; ChromID, ChromID ${ }^{\mathrm{TM}}$ ESBL agar; ${ }^{\mathrm{b}} \mathrm{P}$, positive result; N, positive result.

\subsection{Prospective Evaluation on Routine Antibiograms}

For this study, 102 consecutive expanded-spectrum-cephalosporin-resistant Enterobacterales collected at the Bicêtre hospital from March to July 2018 were included in this study. These bacteria were recovered from urine ( $43 \%$ ), from blood cultures (55\%) and from a bile (2\%) (Table 2). The most prevalent bacteria found in urine and in blood cultures were E. coli $(71 \%)$, and K. pneumoniae $(73 \%)$, respectively. Bacteria were directly tested from a disk diffusion antibiogram on $\mathrm{MH}$ agar. ESBL production was monitored by synergy testing, LFIA using the NG-Test CTX-M MULTI and by homemade-PCR sequencing $[19,20]$. Out of the 102 isolates compatible with ESBL production according to routine antibiogram, 99 were positive using the NG-Test CTX-M MULTI assay (Table 2). Three gave a negative test result, one SHV-12 producing E. cloacae and two hyper producing SHV-28 K. pneumoniae isolates. No invalid test result was observed, no false negative and no false positive results were observed. The results matched perfectly with the PCR-sequencing results. Eighty-one percentage of the ESBLs detected belonged to CTX-M-1 group with CTX-M-15 representing $72 \%$ of the total ESBLs. $16 \%$ of the ESBLs detected belonged to CTX-M-9 group and 3\% were SHV-producers. No plasmid-encoded AmpC ( $\mathrm{pAmpC}$ ) enzymes were detected during this study. 
Table 2. Prospective evaluation of NG-Test CTX-M MULTI.

\begin{tabular}{|c|c|c|c|c|}
\hline Species & Sample Origin & ESBL Identified & $\begin{array}{l}\text { No. of } \\
\text { Isolates }\end{array}$ & $\begin{array}{l}\text { NG-Test CTX-M } \\
\text { MULTI Results }\end{array}$ \\
\hline E. coli & Urine & CTX-M-1 & 4 & $\mathrm{P}$ \\
\hline E. coli & Bile & CTX-M-1 & 1 & $\mathrm{P}$ \\
\hline E. coli & Urine & CTX-M-14 & 6 & $\mathrm{P}$ \\
\hline E. coli & Bile & CTX-M-14 & 1 & $\mathrm{P}$ \\
\hline E. coli & blood & CTX-M-14 & 1 & $\mathrm{P}$ \\
\hline E. coli & Urine & CTX-M-15 & 18 & $\mathrm{P}$ \\
\hline E. coli & blood & CTX-M-15 & 3 & $\mathrm{P}$ \\
\hline E. coli & Urine & CTX-M-27 & 2 & $\mathrm{P}$ \\
\hline E. coli & Urine & CTX-M-32 & 1 & $\mathrm{P}$ \\
\hline E. coli & Urine & CTX-M-55 & 1 & $\mathrm{P}$ \\
\hline E. coli & blood & СТХ-M-9 & 1 & $\mathrm{P}$ \\
\hline K. pneumoniae & blood & CTX-M-14 & 1 & $\mathrm{P}$ \\
\hline K. pneumoniae & blood & CTX-M-15 & 25 & $\mathrm{P}$ \\
\hline K. pneumoniae & Urine & CTX-M-127 & 1 & $\mathrm{P}$ \\
\hline K. pneumoniae & blood & CTX-M-15 & 13 & $\mathrm{P}$ \\
\hline K. pneumoniae & blood & Hyper SHV-28 & 2 & $\mathrm{~N}$ \\
\hline E. cloacae & Urine & СТХ-M-15 & 7 & $\mathrm{P}$ \\
\hline E. cloacae & Urine & СТХ-M-9 & 2 & $\mathrm{P}$ \\
\hline E. cloacae & blood & CTX-M-15 & 6 & $\mathrm{P}$ \\
\hline E. cloacae & blood & CTX-M-3 & 1 & $\mathrm{P}$ \\
\hline E. cloacae & blood & SHV-12 & 1 & $\mathrm{~N}$ \\
\hline C. koseri & Urine & CTX-M-1 & 1 & $\mathrm{P}$ \\
\hline C. koseri & Urine & CTX-M-14 & 1 & $\mathrm{P}$ \\
\hline Salmonella sp. & blood & CTX-M-9 & 1 & $\mathrm{P}$ \\
\hline C. freundii & blood & CTX-M-15 & 1 & $\mathrm{P}$ \\
\hline
\end{tabular}

\subsection{Prospective Evaluation on Blood Culture}

Among the 100 consecutive positive blood cultures with Gram negative Bacilli (GNB), as revealed by gram staining, the NG-Test CTX-M MULTI tested directly without prior sub-culturing using the published protocol by Takissian et al. [23] gave 10 positive results. Routine disk diffusion antibiograms revealed the presence of a phenotype compatible with ESBL production (resistance to expanded-spectrum cephalosporins and recovery by clavulanate) with 11 bacteria grown from these blood cultures. MALDI-TOF MS identification of the bacteria and PCR-sequencing revealed the presence of 5 CTX-M-15-producing E. cloacae, 3 CTX-M-15-producing E. coli, 2 CTX-M-27-producing E. coli and 1 SHV-12-producing E. cloacae). The 10 CTX-M-producing bacteria were from the 10 blood cultures identified as CTX-M-positive by the NG-Test CTX-M MULTI.

\section{Discussion}

CTX-M enzymes are today the most prevalent group of ESBLs among Gram-negative pathogens around the world, representing a global pandemic [7]. There are around 229 CTX-Ms variants described that belong to either CTX-M-1, $-2,-8,-9$ and -25 clusters differing in less than $5 \%$ amino acid sequence within each cluster $[27,28]$, including all plasmid-encoded and the chromosomic variants in Kluyvera species [29]. The global epidemiology of CTX-M variants is complex with some regional specificities [7], but CTX-M-15 is now the most prevalent CTX-M determinant worldwide [7]. Other variants, such as group 9 variants (especially CTX-M-14) are prevalent in China, South-East Asia, South Korea, Japan and Spain, and group 2 and group 8 (CTX-M-2-like and CTX-M-8-like) variants are prevalent in South America [7]. In many European countries, such as in France CTX-M-15 has become the major ESBL encountered in clinical isolates [7]. In our study, 97\% of the ESBLs were CTX-M-like enzymes and $3 \%$ SHVs (one SHV-12 producing E. cloacae and two hyper producing SHV-28 K. pneumoniae isolates). Eighty-one \% of the ESBLs detected belonged to CTX-M-1 group with CTX-M-15 representing $72 \%$ of 
the total ESBLs. $16 \%$ of the ESBLs detected belonged to CTX-M-9 group and 3\% were SHV-producers. No pAmpC enzymes were detected during this study, revealing the low prevalence of theses enzymes in our hospital.

As $b l a_{\mathrm{CTX}}$-M genes are encoded on multidrug-resistant plasmids, treatments of severe infections with CTX-M-producing bacteria rely on carbapenems and are associated with higher rates of healthcare costs, and mortality [1,12]. Rapid identification of CTX-M producers is mandatory for implementation of infection control measures and for initiating rapidly proper treatment [1,12]. Easy-to-carry-out tests, mostly based on inhibition of ESBLs by clavulanic acid or tazobactam, are recommended [30]. A new phenotypic assay that originates from the carbapenem inactivation method (CIM) [31], named the direct $\beta$-lactam inactivation method (dBLIM), that allows detection of extended-spectrum-cephalosporinase activity directly from Enterobacterales in culture or from positive blood cultures, using a 5- $\mu$ gcefotaxime disk [32]. These tests have excellent performances, but their main pitfalls are limited sensitivity, the requirement of an overnight incubation and doesn't give information about the resistance mechanisms or genotype, and thus does not provide any information about the possible threats of spreading.

Detection of ESBLs at the genetic level represents an interesting alternative but remains costly, requires expertise and costly equipment, and may give false positive results due to the detection of inactive or incomplete resistance genes in a specimen [30]. Other techniques, such as matrix-assisted laser desorption ionization-time of flight mass spectrometry (MALDI-TOF MS) [33], are being developed, but they do require expensive equipment, a significant degree of expertise, and the kits are expensive. Finally, biochemical tests have been developed, namely, the Rapid ESBL NDP [34] test, and two commercially available tests: the $\beta$-Lacta test (BioRad), and the Rapid ESBL Screen [35]. The ESBL NDP test and the Rapid ESBL Screen are aimed at detecting ESBL producers, while the $\beta$-Lacta test is aimed at detecting not only ESBL producers but also cephalosporinase- and carbapenemase-producers. The sensitivity and specificity for detecting ESBL producers $(n=60)$ were $95 \%$ and $100 \%$ for the Rapid ESBL NDP test (after $2 \mathrm{~h}$ ), $92 \%$ and $83 \%$ (after $2 \mathrm{~h}$ ) for the Rapid ESBL Screen, and $88 \%$ and $71 \%$ for the $\beta$-Lacta test (after $15 \mathrm{~min}$ ), respectively [36].

The NG-Test CTX-M MULTI detected all the CTX-M-expressing strains without ambiguous interpretation and showed $100 \%$ sensibility and specificity on standard agar containing plates used in clinical microbiology laboratories. Isolates representing the most prevalent variants of the five groups of CTX-Ms were detected, suggesting that this assay may be used on all the continents, irrespective of the prevalence of the different CTX-M variants present.

It is assumed that early identification of ESBL-E bacteraemia may lead to optimized treatment, contributing to better outcomes and early implementation of infection control measures [37]. The ß-Lacta test has been validated for rapid detection of Ambler class A ESBL-E from smudge plates prepared from positive blood cultures [38-40]. Even though the detection of expanded-spectrum cephalosporin (ESC)-resistant Enterobacterales was excellent, it required 2-3 h of growth prior to testing. Alternatively, Dortet et al. has shown that the ESBL NDP test may be able to detect ESBL-E within 30 min after a few centrifugation steps of positive blood cultures [34]. The NG-Test CTX-M MULTI was able to detect 10 out of 11 ESBL-producers in our study in less than $15 \mathrm{~min}$.

Since the first description of Kluyvera as the progenitor of CTX-M-encoded $\beta$-lactamases with no or little phenotypic expression, as compared to their plasmid-borne counterparts [41], chromosomal counterparts belonging to the five sub-groups of acquired CTX-M $\beta$-lactamases have been found in Kluyvera spp. [7,29]. Even if Kluyvera spp. is considered as a benign saprophyte of gastrointestinal, urinary and respiratory tracts, it has been sporadically reported as the cause of clinically significant diseases at multiple anatomic sites, showing an ability to act as an opportunistic pathogen [42,43]. The NG-Test CTX-M MULTI was positive in only 32\% (9/28) of the Kluyvera sp. isolates tested. In one case, the signal was strong, and in 8 cases the signal was weak, which correlates well with the observed resistance phenotype. The detection of the chromosomally encoded CTX-M-variants present 
in Kluyvera spp., is difficult as the expression level of these enzymes in these species is variable and very low [44].

K. oxytoca clinical isolates were also used as controls since they produce structurally related but chromosomally encoded ESBLs (OXY/K1) [45]. Hyperproduction of these chromosomally encoded class A OXY- $\beta$-lactamases confers resistance to aztreonam and expanded spectrum cephalosporins (ESCs) with the exception of ceftazidime and cephamycins, but OXY-2-variants conferring high-level resistance to ceftazidime have also been described [45]. The NG-Test CTX-M MULTI does not cross-react with $K$. oxytoca isolates, unless a plasmid-encoded CTX-M is also produced.

ESBLs, especially CTX-M-type are the main source of cephalosporin resistance in Enterobacterales of human origin in France. The NG-Test CTX-M MULTI is thus well adapted to the French epidemiology, and thus, its routine application of rapid diagnostic tests for ESBL-E detection should lead to improved antimicrobial management, particularly by reducing inappropriate use of carbapenems, especially for bacteraemia. Although these results are promising, they should be further confirmed in other countries where the prevalence and the epidemiology of ESBL-producers might be different and where pAmpC are more prevalent. In addition, more variants need to be tested, even though the variants tested correspond to most prevalent variants worldwide, they represent only 13\% (29/229) of the known variants [28]. Further developments including, TEM-, SHV-, minor-ESBLs and the main pAmpCs (CMY, DHA) would improve the test to reach almost $100 \%$.

\section{Conclusions}

The NG-Test CTX-M MULTI assay detected all the CTX-M-expressing strains without ambiguous interpretation and showed $100 \%$ sensitivity and specificity on standard agar containing plates used in clinical microbiology laboratories. Isolates from the 5 groups of CTX-Ms were detected either from bacterial cultures or directly from positive blood cultures. The NG-Test CTX-M MULTI assay is a commercially-available robust assay, which is stable for $>24$ months without refrigeration, user-friendly (no need for trained staff nor equipment for the readout), rapid (less than $15 \mathrm{~min}$ ) and cost-effective $\sim 7 €$ per test, as compared to commercially available molecular tests (ranging from 20 to $>100$ euros for BioFire-type PCRs). In settings where CTX-Ms are the major source of ESCs resistance, these tests should lead to improved antimicrobial management, particularly by reducing inappropriate use of carbapenems.

Supplementary Materials: The following are available online at http://www.mdpi.com/2075-4418/10/10/764/s1, Table S1: Retrospective evaluation of NG-Test MULTI on well characterized isolates, Table S2: Retrospective evaluation of NG-Test MULTI on K. oxytoca isolates, Table S3: Retrospective evaluation of NG-Test MULTI on Kluyvera spp. isolates.

Author Contributions: Conceptualization, H.V., S.S. and T.N.; methodology, L.D. and T.N.; formal analysis, S.B., K.C.R., C.G. and H.B.; investigation, S.B., K.C.R., A.V., K.D., M.P., S.O., C.G. and H.B; resources, N.F., T.N. and S.M.-K.; writing-original draft preparation, S.B. and T.N.; writing-review and editing, All authors; supervision, H.V., L.D. and T.N.; project administration, H.V. and T.N.; funding acquisition, H.V. and T.N. All authors have read and agreed to the published version of the manuscript.

Funding: This work was supported by the Assistance Publique-Hôpitaux de Paris, the Université Paris Saclay, the Laboratory of Excellence in Research on Medication and Innovative Therapeutics (LERMIT) through a grant from the French National research Agency (ANR-10-LABX-33), by the JPIAMR transnational project DesInMBL (ANR-14-JAMR-0002) and by EIT Health supported projects BL-Detectool and AMR-Detectool.

Acknowledgments: We are grateful to NG Biotech for providing the NG-Test CTX-M MULTI free of charge.

Conflicts of Interest: The authors declare no conflict of interest. The funders had no role in the design of the study; in the collection, analyses, or interpretation of data; in the writing of the manuscript, or in the decision to publish the results. 


\section{References}

1. Paterson, D.L. Resistance in gram-negative bacteria: Enterobacteriaceae. Am. J. Infect. Control. 2006, 34 (Suppl. 1), S20-S28, discussion S64-S73. [CrossRef] [PubMed]

2. Centers for Disease Control and Prevention. Antibiotic Resistance Threats in the United States, 2013; Centers for Disease Control and Prevention: Atlanta, GA, USA, 2014. Available online: https:/www.cdc.gov/drugresistance/ threat-report-2013/index.html (accessed on 25 September 2020).

3. Bauernfeind, A.; Grimm, H.; Schweighart, S. A new plasmidic cefotaximase in a clinical isolate of Escherichia coli. Infection 1990, 18, 294-298. [CrossRef] [PubMed]

4. Bonnet, R. Growing group of extended-spectrum beta-lactamases: TheCTX-Menzymes. Antimicrob. Agents Chemother. 2004, 48, 1-14. [CrossRef] [PubMed]

5. Cantón, R.; Coque, T.M. The CTX-M ß-lactamase pandemic. Curr. Opin. Microbiol. 2006, 9, 466-475. [CrossRef] [PubMed]

6. Cantón, R.; González-Alba, J.M.; Galán, J.C. CTX-M Enzymes: Origin and Diffusion. Front. Microbiol. 2012, 3, 110. [CrossRef]

7. Bevan, E.R.; Jones, A.M.; Hawkey, P.M. Global epidemiology of CTX-M $\beta$-lactamases: Temporal and geographical shifts in genotype. J. Antimicrob. Chemother. 2017, 72, 2145-2155. [CrossRef]

8. Laxminarayan, R.; Duse, A.; Wattal, C.; Zaidi, A.K.; Wertheim, H.F.; Sumpradit, N.; Vlieghe, E.; Hara, G.L.; Gould, I.M.; Goossens, H.; et al. Antibiotic resistance-The need for global solutions. Lancet Infect. Dis. 2013, 13, 1057-1098. [CrossRef]

9. Hawkey, P.M. Multidrug-resistant Gram-negative bacteria: A product of globalization. J. Hosp. Infect. 2015, 89, 241-247. [CrossRef]

10. Tacconelli, E.; Cataldo, M.A.; Dancer, S.J.; De Angelis, G.; Falcone, M.; Frank, U.; Kahlmeter, G.; Pan, A.; Petrosillo, N.; Rodríguez-Baño, J.; et al. ESCMID guidelines for the management of the infection control measures to reduce transmission of multidrug-resistant Gram-negative bacteria in hospitalized patients. Clin. Microbiol. Infect. 2014, 20,1-55. [CrossRef]

11. Ruppé, E.; Hem, S.; Lath, S.; Gautier, V.; Ariey, F.; Sarthou, J.L.; Monchy, D.; Arlet, G. CTX-M $\beta$-Lactamases in Escherichia coli from Community-acquired Urinary Tract Infections, Cambodia. Emerg. Infect. Dis. 2009, 15, 741-748. [CrossRef]

12. D'Andrea, M.M.; Arena, F.; Pallecchi, L.; Rossolini, G.M. CTX-M-type $\beta$-lactamases: A successful story of antibiotic resistance. Int. J. Med. Microbiol. 2013, 303, 305-317. [CrossRef] [PubMed]

13. Nordmann, P.; Dortet, L.; Poirel, L. Carbapenem resistance in Enterobacteriaceae: Here is the storm! Trends Mol. Med. 2012, 18, 263-272. [CrossRef] [PubMed]

14. Krishnamurthy, V. Phenotypic and Genotypic Methods for Detection of Extended Spectrum $\beta$ Lactamase Producing Escherichia coli and Klebsiella pneumoniae Isolated from Ventilator Associated Pneumonia. J. Clin. Diagn. Res. 2013, 7, 1975-1978. [CrossRef] [PubMed]

15. Zamani, K.; Emami, A.; Bazargani, A.; Moattari, A. Phenotypic and molecular characterization of CTX-M extended-spectrum beta-lactamase-producing Escherichia coli isolates in Shiraz, Iran. Rev. Soc. Bras. Med. Trop. 2015, 48, 479-482. [CrossRef]

16. Peker, N.; Rossen, J.W.A.; Deurenberg, R.H.; Langereis, P.C.; Raangs, E.G.C.; Kluytmans, J.A.; Friedrich, A.W.; Veenemans, J.; Sinha, B. Evaluation of an Accelerated Workflow for Surveillance of ESBL (CTX-M)-Producing Escherichia coli Using Amplicon-Based Next-Generation Sequencing and Automated Analysis. Microorganisms 2018, 6, 6. [CrossRef]

17. Naas, T.; Cuzon, G.; Bogaerts, P.; Glupczynski, Y.; Nordmann, P. Evaluation of a DNA microarray (Check-MDR CT102) for rapid detection of TEM, SHV, and CTX-M extended-spectrum $\beta$-lactamases and of KPC, OXA-48, VIM, IMP, and NDM-1 carbapenemases. J. Clin. Microbiol. 2011, 49, 1608-1613. [CrossRef]

18. Girlich, D.; Bernabeu, S.; Fortineau, N.; Dortet, L.; Naas, T. Evaluation of the CRE and ESBL ELITe MGB@kits for the accurate detection of carbapenemase- or CTX-M-producing bacteria. Diagn. Microbiol. Infect. Dis. 2018, 92, 1-7. [CrossRef]

19. Naas, T.; Oxacelay, C.; Nordmann, P. Identification of CTX-M-type extended-spectrum-beta-lactamase genes using real-time PCR and pyrosequencing. Antimicrob. Agents Chemother. 2007, 51, 223-230. [CrossRef]

20. Oxacelay, C.; Ergani, A.; Naas, T.; Nordmann, P. Rapid detection of CTX-M-producing Enterobacteriaceae in urine samples. J. Antimicrob. Chemother. 2009, 64, 986-989. [CrossRef] 
21. Peker, N.; Couto, N.; Sinha, B.; Rossen, J.W. Diagnosis of bloodstream infections from positive blood cultures and directly from blood samples: Recent developments in molecular approaches. Clin. Microbiol. Infect. 2018, 24, 944-955. [CrossRef]

22. De Angelis, G.; Grossi, A.; Menchinelli, G.; Boccia, S.; Sanguinetti, M.; Posteraro, B. Rapid molecular tests for detection of antimicrobial resistance determinants in Gram-negative organisms from positive blood cultures: A systematic review and meta-analysis. Clin. Microbiol. Infect. 2020, 26, 271-280. [CrossRef] [PubMed]

23. Takissian, J.; Bonnin, R.A.; Naas, T.; Dortet, L. NG-Test Carba 5 for Rapid Detection of Carbapenemase-Producing Enterobacterales from Positive Blood Cultures. Antimicrob. Agents Chemother. 2019, 63, e00011-e00019. [CrossRef] [PubMed]

24. Boutal, H.; Naas, T.; Devilliers, K.; Oueslati, S.; Dortet, L.; Bernabeu, S.; Simon, S.; Volland, H. Development and Validation of a Lateral Flow Immunoassay for Rapid Detection of NDM-Producing Enterobacteriaceae. J. Clin. Microbiol. 2017, 55, 2018-2029. [CrossRef] [PubMed]

25. Boutal, H.; Vogel, A.; Bernabeu, S.; Devilliers, K.; Creton, E.; Cotellon, G.; Plaisance, M.; Oueslati, S.; Dortet, L.; Jousset, A.; et al. A multiplex lateral flow immunoassay for the rapid identification of NDM-, KPC-, IMPand VIM-type and OXA-48-like carbapenemase-producing Enterobacteriaceae. J. Antimicrob. Chemother. 2018, 73, 909-915. [CrossRef] [PubMed]

26. Clinical Breakpoints and Dosing of Antibiotics. Available online: https://eucast.org/clinical_breakpoints/ (accessed on 12 August 2020).

27. Naas, T.; Oueslati, S.; Bonnin, R.A.; Dabos, M.L.; Zavala, A.; Dortet, L.; Retailleau, P.; Iorga, B.I. Beta-lactamase database (BLDB)—Structure and function. J. Enzyme Inhib. Med. Chem. 2017, 32, 917-919. [CrossRef]

28. Beta-Lactamase DataBase-Structure and Function. Available online: http://www.bldb.eu/ (accessed on 12 August 2020).

29. Rodriguez, M.M.; Power, P.; Radice, M.; Vay, C.; Famiglietti, A.; Galleni, M.; Ayala, J.A.; Gutkind, G. Chromosome-encoded CTX-M-3 from Kluyvera ascorbata: A possible origin of plasmid-borne CTX-M-1-derived cefotaximases. Antimicrob. Agents Chemother. 2004, 48, 4895-4897. [CrossRef]

30. Gazin, M.; Paasch, F.; Goossens, H.; Malhotra-Kumar, S. MOSAR WP2 and SATURN WP1 Study Teams. Current trends in culture-based and molecular detection of extended-spectrum- $\beta$-lactamase-harboring and carbapenem-resistant Enterobacteriaceae. J. Clin. Microbiol. 2012, 50, 1140-1146. [CrossRef]

31. van der Zwaluw, K.; de Haan, A.; Pluister, G.N.; Bootsma, H.J.; de Neeling, A.J.; Schouls, L.M. The carbapenem inactivation method (CIM), a simple and low-cost alternative for the Carba NP test to assess phenotypic carbapenemase activity in gram-negative rods. PLoS ONE 2015, 10, e0123690. [CrossRef]

32. Bianco, G.; Boattini, M.; Iannaccone, M.; Fossati, L.; Cavallo, R.; Costa, C. Direct $\beta$-Lactam Inactivation Method: A New Low-Cost Assay for Rapid Detection of Carbapenemase- or Extended-Spectrum- $\beta$-Lactamase-Producing Enterobacterales Directly from Positive Blood Culture Bottles. J. Clin. Microbiol. 2019, 58, e01178-19. [CrossRef]

33. Sparbier, K.; Schubert, S.; Weller, U.; Boogen, C.; Kostrzewa, M. Matrix-assisted laser desorption ionization-time of flight mass spectrometry-based functional assay for rapid detection of resistance against $\beta$-lactam antibiotics. J. Clin. Microbiol. 2012, 50, 927-937. [CrossRef]

34. Dortet, L.; Poirel, L.; Nordmann, P. Rapid detection of extended-spectrum- $\beta$-lactamase-producing Enterobacteriaceae in blood cultures. Emerg. Infect. Dis. 2014, 21, 504-507. [CrossRef] [PubMed]

35. Renvoisé, A.; Decré, D.; Amarsy-Guerle, R.; Huang, T.D.; Jost, C.; Podglajen, I.; Raskine, L.; Genel, N.; Bogaerts, P.; Jarlier, V.; et al. Evaluation of the $\beta$-Lacta test, a rapid test detecting resistance to third-generation cephalosporins in clinical strains of Enterobacteriaceae. J. Clin. Microbiol. 2013, 51, 4012-4017. [CrossRef] [PubMed]

36. Poirel, L.; Fernández, J.; Nordmann, P. Comparison of Three Biochemical Tests for Rapid Detection of Extended-Spectrum- $\beta$-Lactamase-Producing Enterobacteriaceae. J. Clin. Microbiol. 2016, 54, 423-427. [CrossRef] [PubMed]

37. Goodman, K.E.; Lessler, J.; Cosgrove, S.E.; Harris, A.D.; Lautenbach, E.; Han, J.H.; Milstone, A.M.; Massey, C.J.; Tamma, P.D. A clinical decision tree to predict whether a bacteremic patient is infected with an extended-spectrum $\beta$-lactamase-producing organism. Clin. Infect. Dis. 2016, 63, 896-903. [CrossRef]

38. Chen, Y.; Porter, V.; Mubareka, S.; Kotowich, L.; Simor, A.E. Rapid identification of bacteria directly from positive blood cultures by use of a serum separator tube, smudge plate preparation, and matrix-assisted laser desorption ionization-time of flight mass spectrometry. J. Clin. Microbiol. 2015, 53, 3349-3352. [CrossRef] 
39. Hasso, M.; Porter, V.; Simor, A.E. Evaluation of the $\beta$-Lacta Test for Detection of Extended-Spectrum- $\beta$-Lactamase (ESBL)-Producing Organisms Directly from Positive Blood Cultures by Use of Smudge Plates. J. Clin. Microbiol. 2017, 55, 3560-3562. [CrossRef]

40. Compain, F.; Bensekhri, H.; Rostane, H.; Mainardi, J.L.; Lavollay, M. B-LACTA test for rapid detection of Enterobacteriaceae resistant to third-generation cephalosporins from positive blood cultures using briefly incubated solid medium cultures. J. Med. Microbiol. 2015, 64, 1256-1259. [CrossRef]

41. Humeniuk, C.; Arlet, G.; Gautier, V.; Grimont, P.; Labia, R.; Philippon, A. B-Lactamases of Kluyvera ascorbata, probable progenitors of some plasmid-encoded CTX-M types. Antimicrob. Agents Chemother. 2002, 46, 3045-3049. [CrossRef]

42. Luttrell, R.E.; Rannick, G.A.; Soto-Hernandez, J.L.; Verghese, A. Kluyvera species soft tissue infection: Case report and review. J. Clin. Microbiol. 1988, 26, 2650-2651. [CrossRef]

43. Karadag Oncel, E.; Ozsurekci, Y.; Akyon, Y.; Gur, D.; Cengiz, A.B.; Kara, A. Kluyvera ascorbata infections in children: A case series. Turk. Pediatri Ars. 2015, 50, 123-128. [CrossRef]

44. Lartigue, M.F.; Poirel, L.; Aubert, D.; Nordmann, P. In vitro analysis of ISEcp1B-mediated mobilization of naturally occurring beta-lactamase gene blaCTX-M of Kluyvera ascorbata. Antimicrob. Agents Chemother. 2006, 50, 1282-1286. [CrossRef] [PubMed]

45. Nijhuis, R.H.; Oueslati, S.; Zhou, K.; Bosboom, R.W.; Rossen, J.W.; Naas, T. OXY-2-15, a novel variant showing increased ceftazidime hydrolytic activity. J. Antimicrob. Chemother. 2015, 70, 1429-1433. [CrossRef] [PubMed]

(C) 2020 by the authors. Licensee MDPI, Basel, Switzerland. This article is an open access article distributed under the terms and conditions of the Creative Commons Attribution (CC BY) license (http://creativecommons.org/licenses/by/4.0/). 